\title{
Chalcogen-Bonding Supramolecular Polymers
}

Rongjin Zeng, Zehao Gong, and Qiang Yan*

State Key Laboratory of Molecular Engineering of Polymers, Department of Macromolecular Science, Fudan University, Shanghai 200433, China

\section{Table of Contents}

Figure S1: Photographs of TeA[4] and TeA[4]/DBN complex solution...............page S2

Figure S2: UV-vis change of TeA[4] and TeA[4]/DBN complexation................page S2

Figure S3: ${ }^{1} \mathrm{H}$ NMR chemical shift of TeA[4]/DBN vs. concentration................page S3

Table S1: Calculated parameters of TeA[4]/DBN supramolecular polymerization....page S4

Figure S4: ${ }^{125} \mathrm{Te}$ NMR change of TeA[4]/DBN vs. concentration.....................page S5

Figure S5: DOSY NMR change of TeA[4]/DBN vs. concentration.................page S6

Figure S6: AF4 traces of TeA[4] and TeA[4]/DBN complex.......................page S7

Figure S7: Photographs of SeA[4] and $\mathrm{SeA}[4] / \mathrm{DBN}$ complex solution...............page S8

Figure S8: UV-vis change of $\mathrm{SeA}[4]$ and $\mathrm{SeA}[4] / \mathrm{DBN}$ complexation................page S8

Figure S9: ${ }^{1} \mathrm{H}$ NMR chemical shift of $\mathrm{SeA}[4] / \mathrm{DBN}$ vs. concentration............... page S9

Table S2: Calculated parameters of SeA[4]/DBN supramolecular polymerization...page S10

Figure S10: ${ }^{77} \mathrm{Te}$ NMR change of $\mathrm{SeA}[4] / \mathrm{DBN}$ vs. concentration...................page S11

Figure S11: Rheological comparison of TeA[4]/DBN and SeA[4]/DBN.............page S12

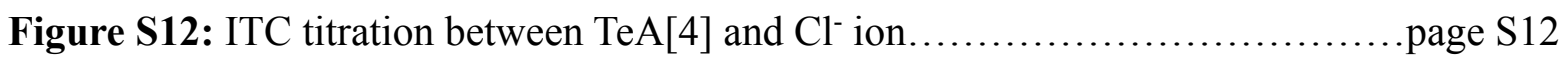

Figure S13: Photographs of reversible dissociation of TeA[4]/DBN hydrogel.......page S13

Figure S14: ${ }^{1} \mathrm{H}$ NMR spectra of 1,3-bis(1,2,5-telluradiazol-1-yl)anisole..............page S17

Figure S15: ${ }^{13} \mathrm{C}$ NMR spectra of 1,3-bis(1,2,5-telluradiazol-1-yl)anisole.............page S17

Figure S16: ${ }^{1} \mathrm{H}$ NMR spectra of 1,3-bis(1,2,5-selenadiazol-1-yl)anisole..............page S18

Figure S17: ${ }^{13} \mathrm{C}$ NMR spectra of 1,3-bis(1,2,5-selenadiazol-1-yl)anisole.............page S18

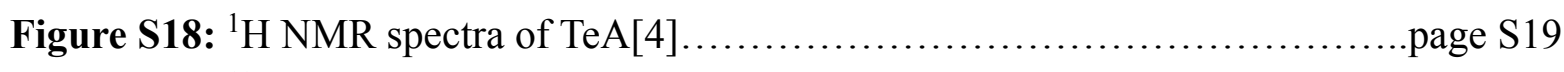

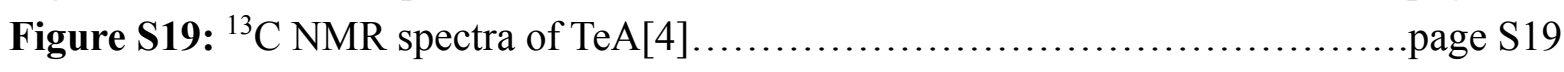

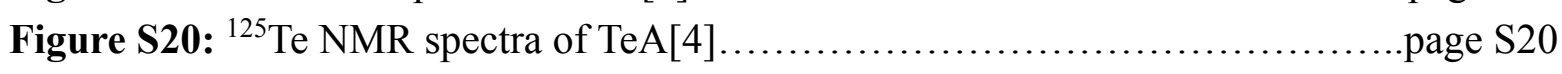

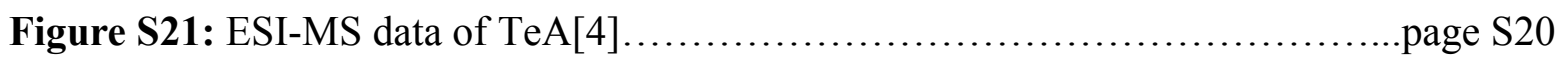

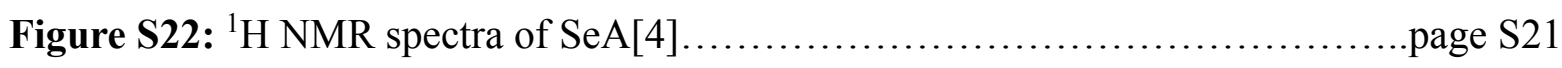

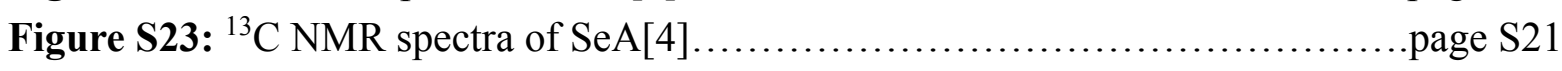

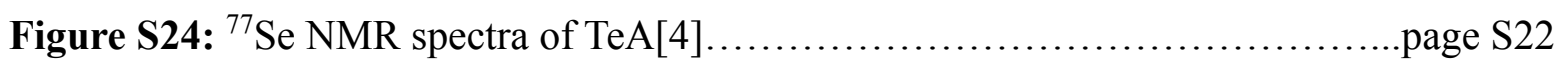

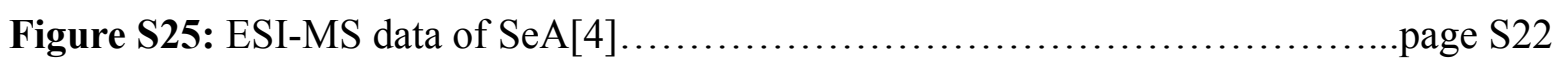




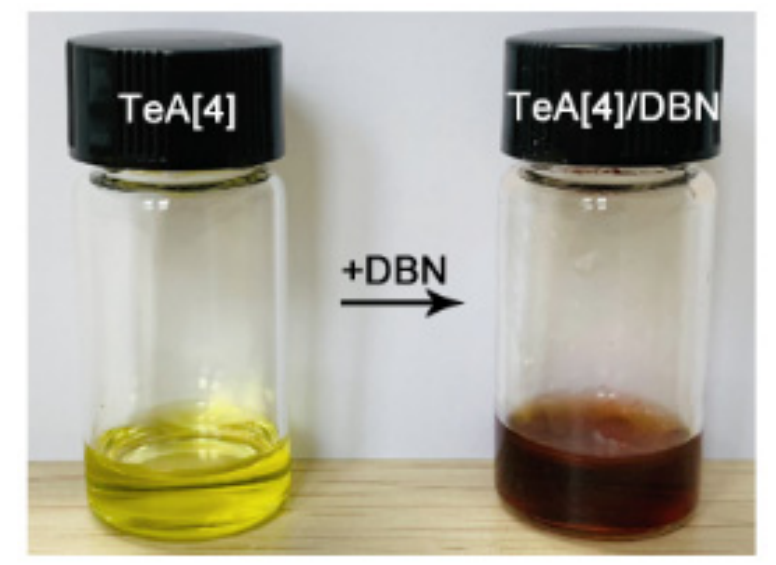

Figure S1. Photographs of TeA[4] solution $\left(10 \mathrm{mM}, \mathrm{CH}_{3} \mathrm{CN}\right)$ and $\mathrm{Te} A[4] / \mathrm{DBN}$ binary solution (10 mM, $\mathrm{CH}_{3} \mathrm{CN} /$ water, 1/1, v/v). The single TeA[4] solution has a light yellow color whereas the equimolar ratio of $\mathrm{Te} A[4] / \mathrm{DBN}$ solution changes to the scarlet color, which suggests a strong chalcogen-bonding-induced charge transfer effect.

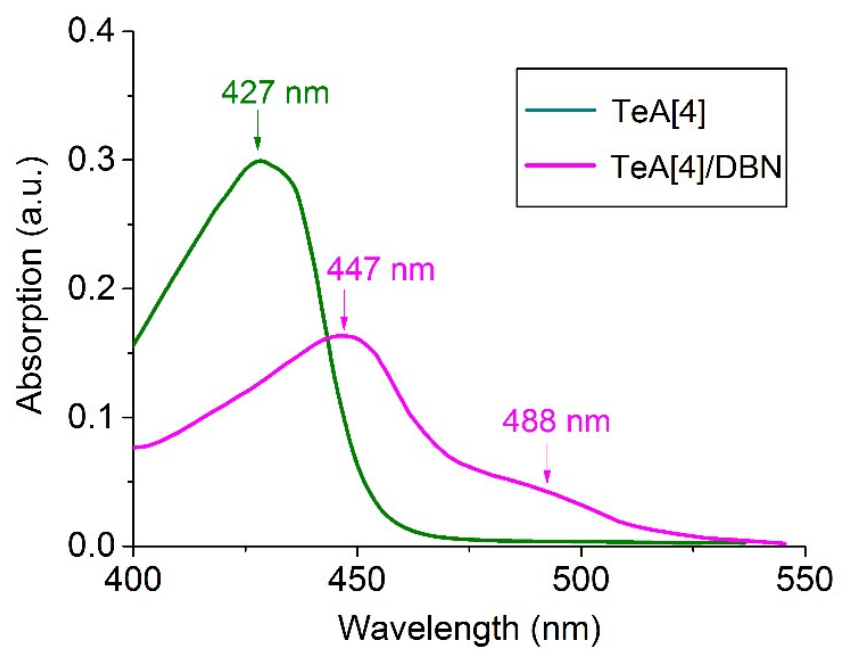

Figure S2. The noncovalent complexation process confirmed by UV-Vis spectroscopy. TeA[4] macrocycle compound $(10 \mathrm{mM})$ showing a characteristic absorption at $\lambda_{\max }=427 \mathrm{~nm}$ (green curve); TeA[4]/DBN solution (1:1) showing a red-shift to $447 \mathrm{~nm}$ and accompanying with a broad long-wavelength absorption band at $488 \mathrm{~nm}$ (pink curve), indicating formation of chalcogen-bonding-induced charge-transfer complex. 


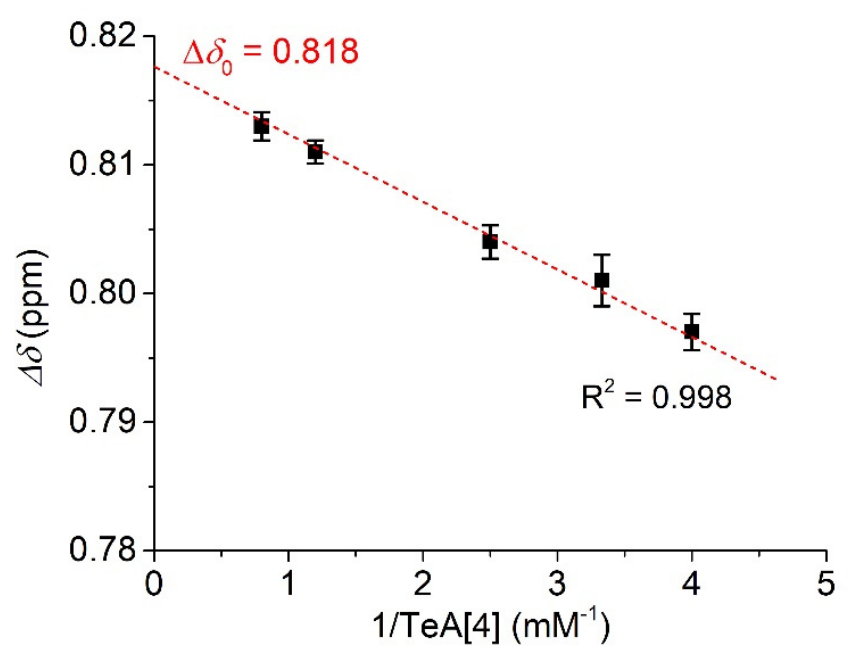

Figure S3. Determination of $\Delta \delta_{0}$ of $\mathrm{H}^{3}$ for TeA[4] and DBN in $\mathrm{CD}_{3} \mathrm{CN}(\mathrm{TeA}[4] / \mathrm{DBN}=1: 1)$ from the extrapolated curve of the ${ }^{1} \mathrm{H}$ NMR spectra. The calculated $\Delta \delta_{0}=0.818 \mathrm{ppm}$.

Based on the Carothers equation, ${ }^{1,2}$ in a typical supramolecular polymerization process, the monomeric complexed species $(p)$ and the degree of polymerization $(n)$ is calculated by $n=$ $1 /(1-p)$, where the $p$ value can be obtained from the chemical shifts in proton NMR spectra. In general, one can select a proton peak as the standard reference, the proportion between the chemical shift value $(\Delta \delta)$ of this proton with the supramolecular polymerization process and its extrapolated value $\left(\Delta \delta_{0}\right)$ is defined as the $p=\Delta \delta / \Delta \delta_{0}$. According to the ${ }^{1} \mathrm{H}$ NMR spectral changes as the concentration increased, if we used $\mathrm{H}^{3}$ of $\mathrm{Te} \mathrm{A}[4]$ as the reference, the actual supramolecular polymerization parameters can be obtained. 
Table S1. Calculated values of the complexed species $(p)$ and polymerization degree $(n)$ at different initial concentrations of TeA[4] and DBN.

\begin{tabular}{cccc}
\hline TeA[4] (mM) & $\Delta \delta(\mathrm{ppm})$ & $p^{\mathbf{a}}$ & $n^{\mathbf{b}}$ \\
\hline 30 & 0.789 & 0.965 & 29 \\
60 & 0.793 & 0.969 & 33 \\
90 & 0.801 & 0.979 & 48 \\
120 & 0.806 & 0.985 & 67 \\
150 & 0.812 & 0.992 & 125 \\
\hline
\end{tabular}

${ }^{a} p=\Delta \delta / \Delta \delta_{0}, \Delta \delta_{0}$ of $\mathrm{H}^{3}$ proton shift was determined to be $0.818 \mathrm{ppm}$ by extrapolation of a plot of $\Delta \delta$ versus $1 / T e A[4]$ at constant DBN.

${ }^{b} n=1 /(1-p), n$ was calculated by Carothers equation. 


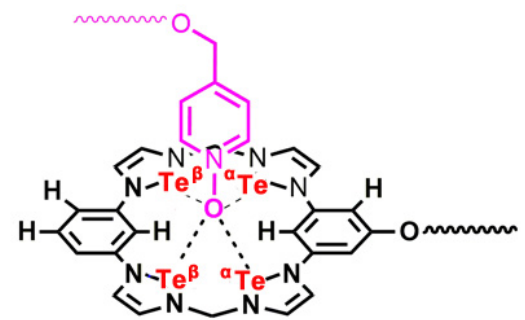

(vi)

$$
\text { (v) }
$$

(iv)

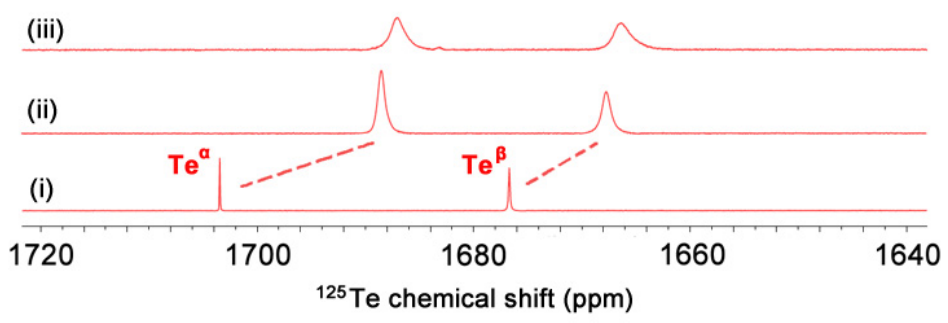

Figure S4. ${ }^{125} \mathrm{Te}$ NMR spectra showing the chalcogen-bonding-driven supramolecular polymerization between TeA[4] and DBN: i) single TeA and ii-vi) equimolar solution of TeA[4] and DBN at concentrations of ii) 30 , iii) 60 , iv) 90 , v) 120 , and vi) $150 \mathrm{mM}$. 


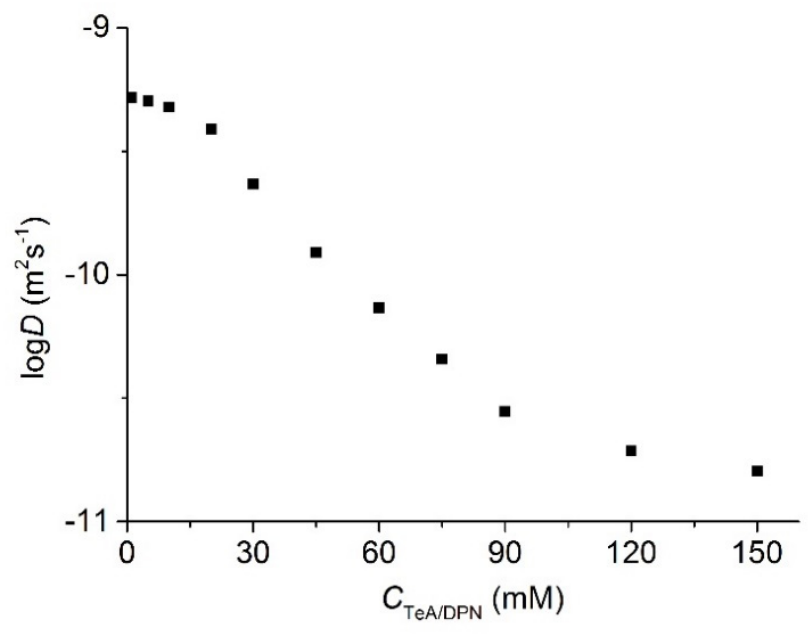

Figure S5. Concentration dependence of diffusion coefficient $D$ plotted against TeA[4] and DBN concentration determined by DOSY NMR. 


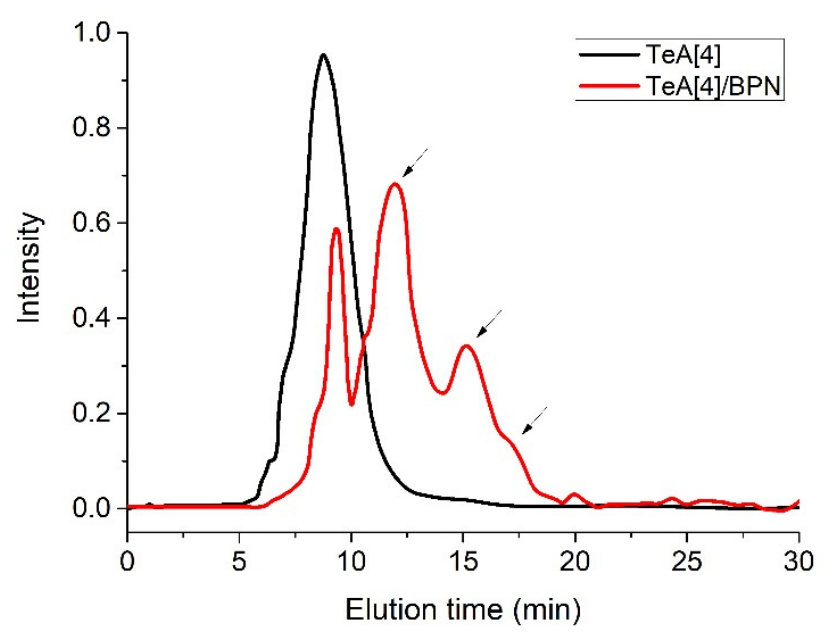

Figure S6. AF4 traces showing the TeA[4] (black curve) and the TeA[4]/BPN complex (red curve): It can be seen that the TeA[4] macrocyclic compound exhibits a single-peak of molecular weight of $M_{\mathrm{w}}=2.2 \times 10^{3} \mathrm{~g} / \mathrm{mol}$, whereas the AF4 profile of TeA[4] $/ \mathrm{BPN}$ mixture in equimolar ratio $(150 \mathrm{mM})$ appears a multiple peak pattern and shifts to the higher elution time. The different peaks corresponds to the $M_{\mathrm{w}}$ of $7.2 \times 10^{3} \mathrm{~g} / \mathrm{mol}, 1.69 \times 10^{4} \mathrm{~g} / \mathrm{mol}$ and $2.82 \times 10^{4}$ $\mathrm{g} / \mathrm{mol}$, in line with the short oligomers. This result indicates that the anionic ligand without long flexible spacer is difficult to sustain a long chalcogen-bonding supramolecular polymer. 


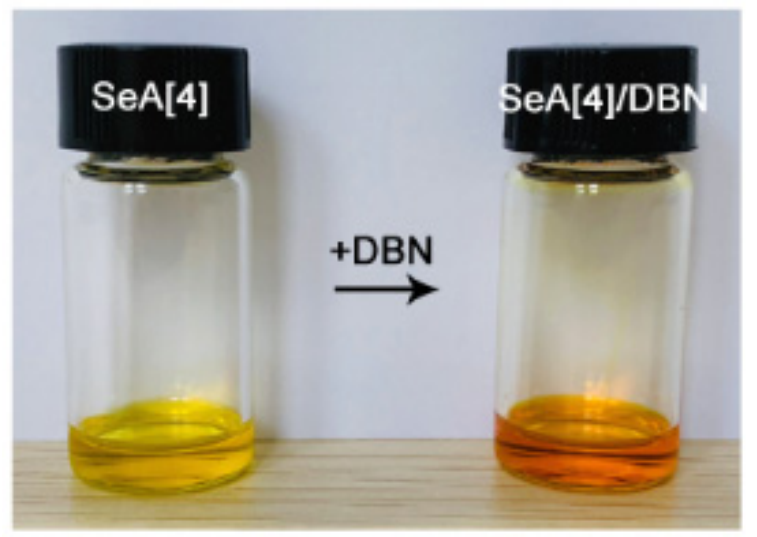

Figure S7. Photographs of $\mathrm{SeA}[4]$ solution $\left(10 \mathrm{mM}, \mathrm{CH}_{3} \mathrm{CN}\right)$ and $\mathrm{SeA}[4] / \mathrm{DBN}$ binary solution (10 mM, $\mathrm{CH}_{3} \mathrm{CN} /$ water, 1/1, v/v). The single $\mathrm{SeA}[4]$ solution has a light yellow color whereas the equimolar ratio of $\mathrm{SeA}[4] / \mathrm{DBN}$ solution changes to orange-red color. This clear color transition suggests a moderate chalcogen-bonding charge transfer effect between SeA[4] macrocyclic receptor and DBN ligand.

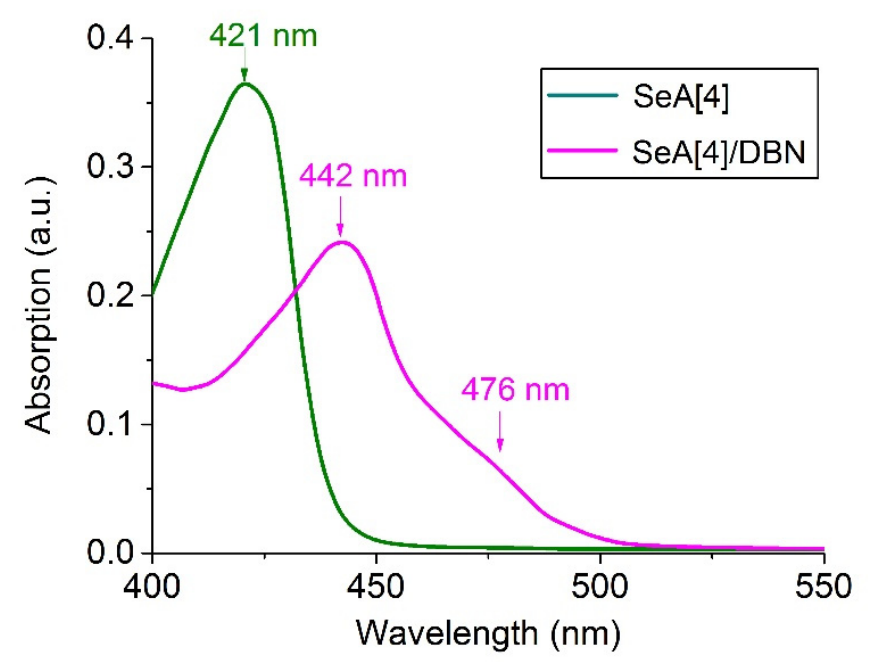

Figure S8. The noncovalent complexation process confirmed by UV-Vis spectroscopy. SeA[4] macrocycle compound $(10 \mathrm{mM})$ showing a characteristic absorption at $\lambda_{\max }=421 \mathrm{~nm}$ (green curve); SeA[4]/DBN solution (1:1) showing a red-shift to $442 \mathrm{~nm}$ and accompanying with a broad long-wavelength absorption band at $476 \mathrm{~nm}$ (pink curve), indicating formation of chalcogen-bonding-induced charge-transfer complex. 


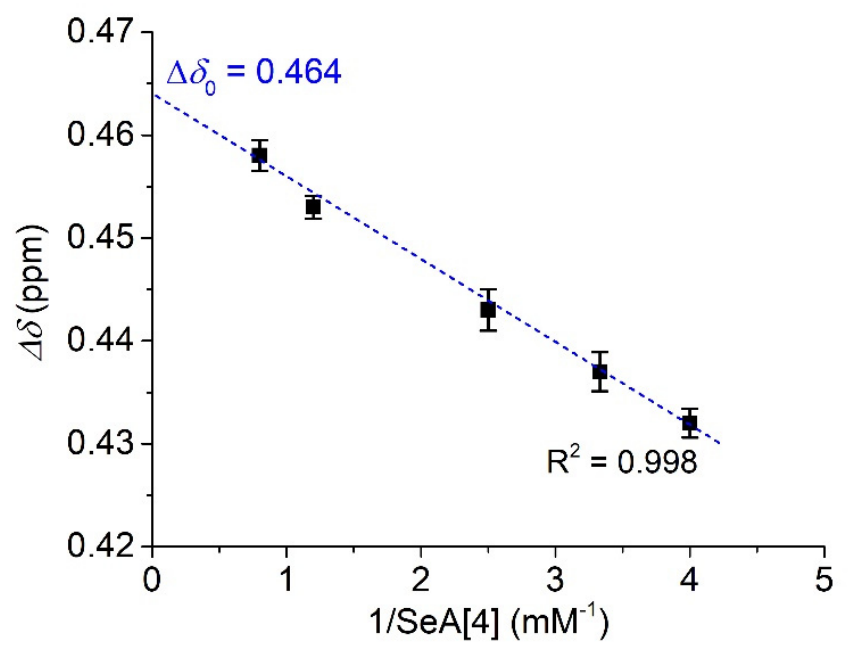

Figure S9. Determination of $\Delta \delta_{0}$ of $\mathrm{H}^{3}$ for $\mathrm{SeA}[4]$ and $\mathrm{DBN}$ in $\mathrm{CD}_{3} \mathrm{CN}(\mathrm{SeA}[4] / \mathrm{DBN}=1: 1)$ from the extrapolated curve. The calculated $\Delta \delta_{0}=0.818 \mathrm{ppm}$. Using the same method, the $p$ and $n$ value can be also calculated by Carothers equation. For example, at $150 \mathrm{mM}, p$ is determined to be 0.982 and $n$ of 56 , corresponding to a SeA[4]/DBN polymer with molar mass of $113 \mathrm{kDa}$; this value is much lower than that of TeA[4]/DBN at the same concentration $(n=$ 125 and $M_{\mathrm{w}}=301 \mathrm{kDa}$ ), indicating that the $\mathrm{Se}^{\cdots} \mathrm{O}^{-}$chalcogen-bonding strength is inferior to that of $\mathrm{Te}^{\cdots} \mathrm{O}^{-}$bonding. 
Table S2. Calculated values of the complexed species $(p)$ and polymerization degree $(n)$ at different initial concentrations of SeA[4] and DBN.

\begin{tabular}{cccc}
\hline $\operatorname{SeA}[4](\mathrm{mM})$ & $\Delta \delta(\mathrm{ppm})$ & $\boldsymbol{p}^{\mathbf{a}}$ & $\boldsymbol{n}^{\mathbf{b}}$ \\
\hline 30 & 0.438 & 0.943 & 18 \\
60 & 0.442 & 0.952 & 21 \\
90 & 0.444 & 0.956 & 23 \\
120 & 0.451 & 0.971 & 34 \\
150 & 0.456 & 0.982 & 56 \\
\hline
\end{tabular}

${ }^{a} p=\Delta \delta / \Delta \delta_{0}, \Delta \delta_{0}$ of $\mathrm{H}^{3}$ proton shift was determined to be $0.464 \mathrm{ppm}$ by extrapolation of a plot of $\Delta \delta$ versus $1 / \mathrm{SeA}[4]$ at constant DBN.

${ }^{b} n=1 /(1-p), n$ was calculated by Carothers equation. 


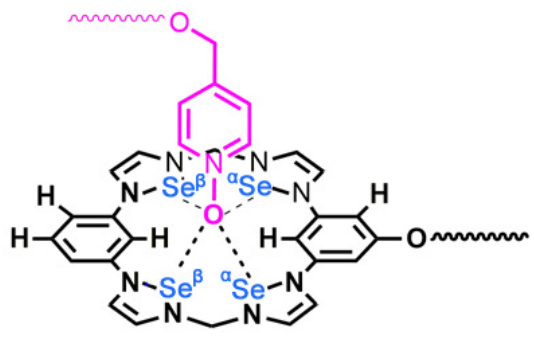

(vi)

(v)

(iv)

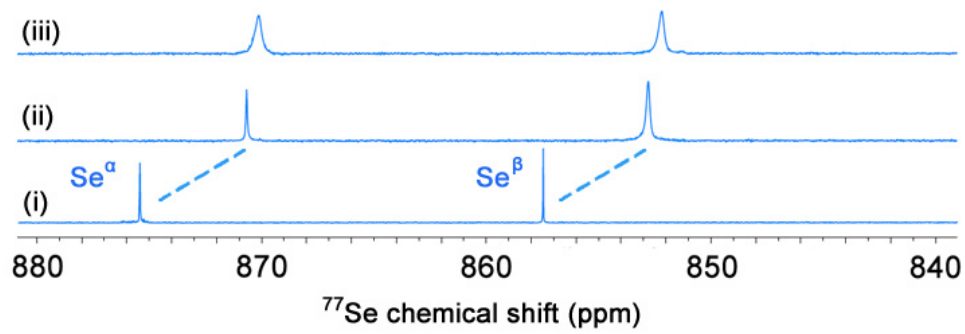

Figure S10. ${ }^{77}$ Se NMR spectra showing the chalcogen-bonding-driven supramolecular polymerization between $\mathrm{SeA}[4]$ and $\mathrm{DBN}$ : i) SeA and ii-vi) equimolar solution of $\mathrm{SeA}[4]$ and DBN at concentrations of ii) 30 , iii) 60 , iv) 90 , v) 120 , and vi) $150 \mathrm{mM}$. 


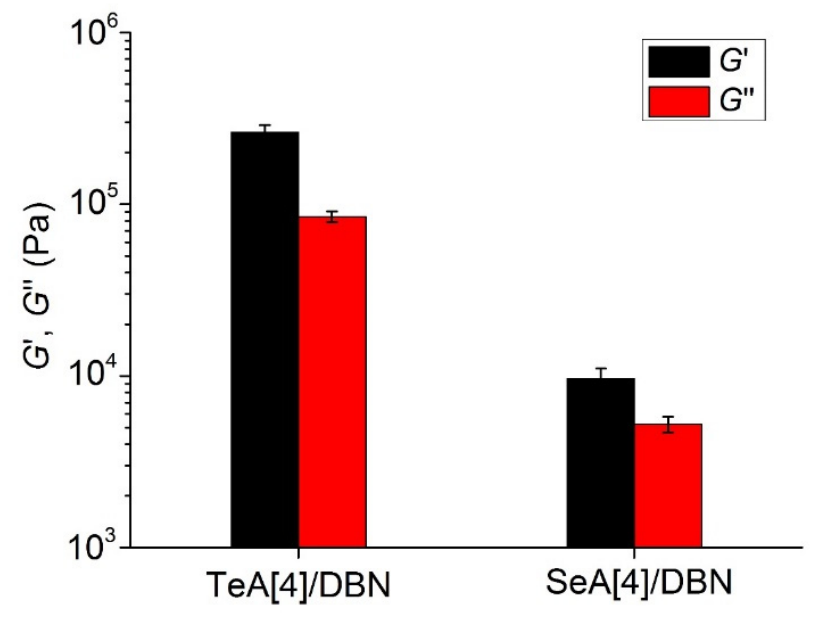

Figure S11. Rheology data comparison of different chalcogen-bonding supramolecular polymer hydrogels (TeA[4]/DBN, left; SeA[4]/DBN, right). It is clear that the storage modulus $\left(G^{\prime}\right)$ of $\mathrm{Te}$ [4] $/ \mathrm{DBN}$ supramolecular hydrogel at $150 \mathrm{mM}$ can reach $\sim 260 \mathrm{kPa}$, whereas

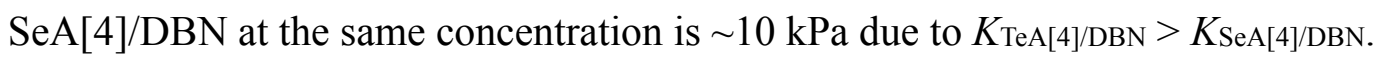

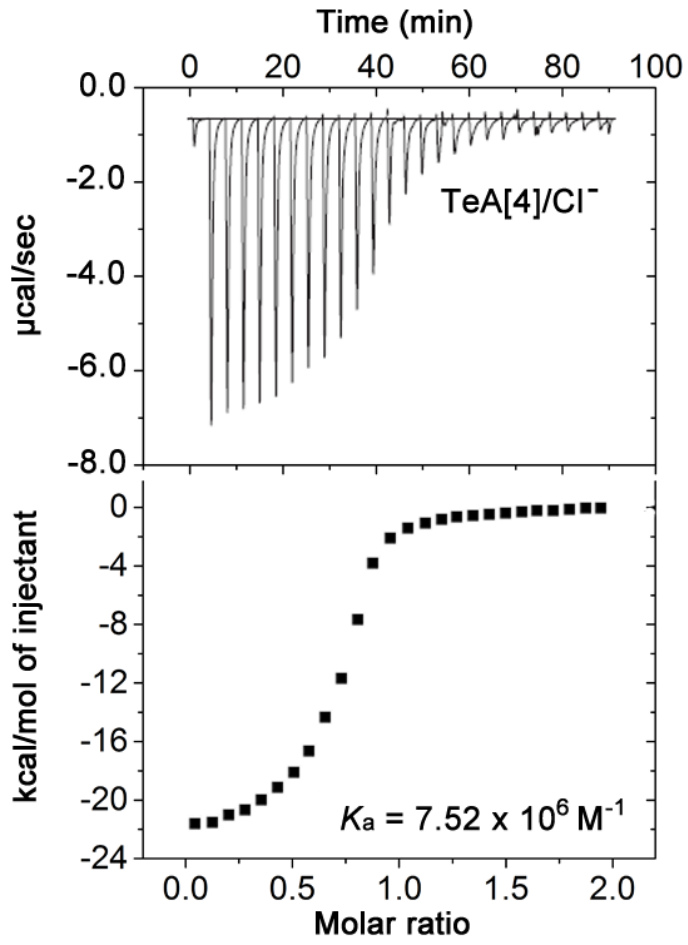

Figure S12. ITC titration showing the stronger binding affinities of $\mathrm{Te} \mathrm{A}[4] / \mathrm{Cl}^{-}\left(\mathrm{Ka}_{\mathrm{a}}=\right.$ $\left.7.52 \times 10^{6} \mathrm{M}^{-1}\right)$. 

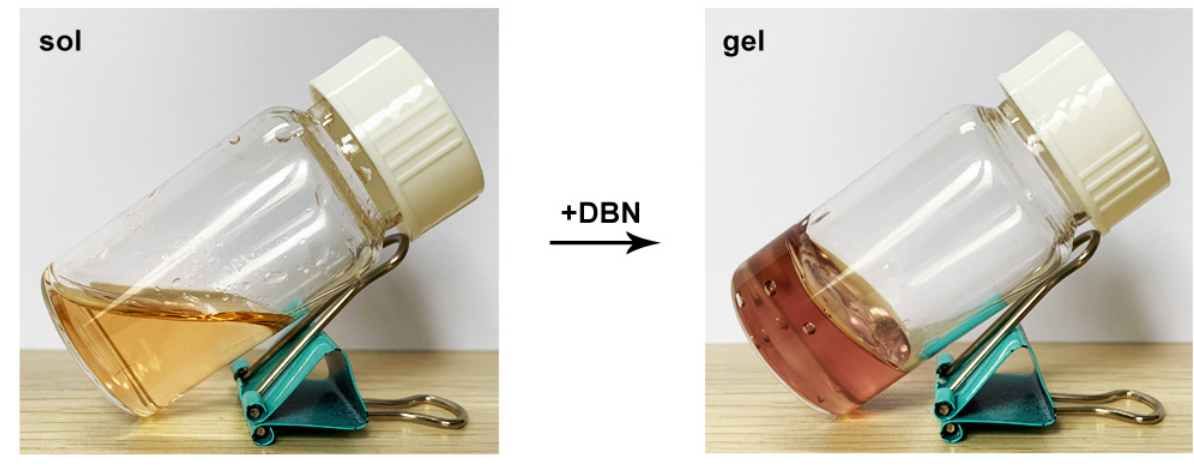

Figure S13. Reversible reconstruction of TeA[4]/DBN chalcogen-bonding hydrogels via addition of excess of DBN monomer. 


\section{DFT Calculations.}

DFT calculations were performed to demonstrate the molecular geometry of TeA and SeA[4] as a chalcogen bonding donor.

The Cartesian coordinate of TeA[4]:

$\begin{array}{llll}\mathrm{N}(1) & -7.3234 & 4.0117 & 0.1333 \\ \mathrm{C}(2) & -6.2331 & 4.9510 & 0.3881 \\ \mathrm{~N}(3) & -5.0553 & 4.0762 & 0.6165 \\ \mathrm{C}(4) & -8.3214 & 4.2860 & -0.6476 \\ \mathrm{C}(5) & -9.04663 .3128 & -1.1406 \\ \mathrm{~N}(6) & -8.85402 .1191 & -0.8551 \\ \mathrm{Te}(7) & -7.7661 & 2.1819 & 0.9117 \\ \mathrm{Te}(8) & -4.0785 & 2.9057 & -0.7553 \\ \mathrm{~N}(9) & -3.1657 & 2.3392 & 1.0057 \\ \mathrm{C}(10) & -3.2448 & 3.3513 & 1.8019 \\ \mathrm{C}(11) & -4.2131 & 4.2420 & 1.6230 \\ \mathrm{C}(12) & -9.3098 & 1.0227 & -1.3237 \\ \mathrm{C}(13) & -2.3998 & 1.3216 & 1.0650 \\ \mathrm{C}(14) & -10.4731 & 1.0660 & -2.0224 \\ \mathrm{C}(15) & -10.9748 & -0.0954 & -2.4848 \\ \mathrm{C}(16) & -10.3482 & -1.2727 & -2.2613 \\ \mathrm{C}(17) & -9.1978 & -1.3614 & -1.5727 \\ \mathrm{C}(18) & -8.6726 & -0.1594 & -1.2293 \\ \mathrm{~N}(19) & -8.4726 & -2.3970 & -1.4030 \\ \mathrm{C}(20) & -2.8677 & 0.1481 & 0.6378 \\ \mathrm{C}(21) & -2.1928 & -1.0208 & 0.6977 \\ \mathrm{C}(22) & -0.9912 & -0.8778 & 1.2591 \\ \mathrm{C}(23) & -0.4951 & 0.2384 & 1.8168 \\ \mathrm{C}(24) & -1.2368 & 1.3441 & 1.6996 \\ \mathrm{~N}(25) & -2.6726 & -2.0932 & 0.2499 \\ \mathrm{Te}(26) & -4.0351 & -2.1233 & -1.2846 \\ \mathrm{~N}(27) & -4.1992 & -4.0445 & -0.6131 \\ \mathrm{C}(28) & -3.1261 & -4.3692 & -0.0236 \\ \mathrm{C}(29) & -2.2913 & -3.3357 & 0.3693 \\ \mathrm{C}(30) & -8.5014 & -3.5240 & -2.0030 \\ \mathrm{C}(31) & -7.5430 & -4.4656 & -1.8577 \\ \mathrm{~N}(32) & -6.5808 & -4.2698 & -1.0097 \\ \mathrm{Te}(33) & -7.4159 & -2.8599 & 0.3177 \\ \mathrm{C}(34) & -5.2968 & -5.0255 & -0.8527 \\ \mathrm{C}(35) & 0.9130 & 0.1982 & 2.4192 \\ \mathrm{H}(36) & -6.5351 & 5.5855 & 1.3029 \\ \mathrm{H}(37) & -6.0750 & 5.6118 & -0.4879 \\ \mathrm{H}(38) & -8.3754 & 5.3956 & -0.8980 \\ \mathrm{H}(39) & -9.8183 & 3.6798 & -1.7862 \\ & & & \end{array}$




$$
\begin{array}{llll}
\mathrm{H}(40) & -2.6664 & 3.3886 & 2.7017 \\
\mathrm{H}(41) & -4.3499 & 5.0037 & 2.4237 \\
\mathrm{H}(42) & -11.1876 & 1.9537 & -2.1486 \\
\mathrm{H}(43) & -12.0181 & -0.1476 & -2.9510 \\
\mathrm{H}(44) & -10.8147 & -2.1381 & -2.7433 \\
\mathrm{H}(45) & -7.6712 & -0.1526 & -0.7783 \\
\mathrm{H}(46) & -3.9086 & 0.1661 & 0.4550 \\
\mathrm{H}(47) & -0.2788 & -1.7077 & 1.3645 \\
\mathrm{H}(48) & -0.9242 & 2.3328 & 2.1014 \\
\mathrm{H}(49) & -2.9513 & -5.4916 & 0.2067 \\
\mathrm{H}(50) & -1.3550 & -3.5064 & 0.8817 \\
\mathrm{H}(51) & -9.3901 & -3.6972 & -2.6199 \\
\mathrm{H}(52) & -7.6345 & -5.4684 & -2.4079 \\
\mathrm{H}(53) & -5.0035 & -5.6190 & -1.7485 \\
\mathrm{H}(54) & -5.2641 & -5.6716 & 0.0928 \\
\mathrm{H}(55) & 1.0751 & 1.1494 & 2.9370 \\
\mathrm{H}(56) & 0.9952 & -0.6316 & 3.1915 \\
\mathrm{H}(57) & 1.6995 & 0.1442 & 1.6319
\end{array}
$$

The Cartesian coordinate of $\mathrm{SeA}[4]$ :
$\mathrm{N}(1) \quad-7.5416 \quad 3.8159 \quad-0.5552$
C(2) $\quad-6.37394 .7206-0.5102$
$\mathrm{N}(3) \quad-5.3108 \quad 4.0153 \quad 0.0877$
C(4) $\quad-8.68684 .2107 \quad-0.9202$
C(5) $\quad-9.6262 \quad 3.2584 \quad-1.0247$
$\mathrm{N}(6) \quad-9.2308 \quad 2.0552 \quad-0.9002$
$\operatorname{Se}(7) \quad-7.78892 .1672 \quad 0.2465$
$\begin{array}{llll}\operatorname{Se}(8) & -4.2439 & 2.7230 & -0.6740\end{array}$
$\mathrm{N}(9) \quad-3.8282 \quad 2.3259 \quad 1.0824$
$\begin{array}{llll}C(10) & -3.9784 & 3.4256 & 1.7425\end{array}$
C(11) $\quad-4.8081 \quad 4.3744 \quad 1.1907$
C(12) $\quad-9.79710 .9186 \quad-0.9322$
C(13) $\quad-3.2240 \quad 1.2459 \quad 1.4452$
C(14) $\quad-11.11790 .7011-0.7448$
$\mathrm{C}(15) \quad-11.6036-0.5236-0.7880$
C(16) - $-10.7789-1.5810-0.9877$
C(17) -9.4633 -1.4219-1.1134
C(18) $\quad-9.0010-0.1353-1.1270$
$\mathrm{N}(19) \quad-8.6451 \quad-2.4049-1.3001$
$\begin{array}{lllll}\mathrm{C}(20) & -3.4382 & 0.0514 & 0.8197\end{array}$
$\mathrm{C}(21) \quad-2.8688-1.1172 \quad 1.1917$
$C(22) \quad-2.0590 \quad-1.0018 \quad 2.2535$
$\mathrm{C}(23) \quad-1.8116 \quad 0.1274 \quad 2.9096$
$\begin{array}{lllll}C(24) & -2.3933 & 1.2404 & 2.4927\end{array}$ 


$$
\begin{array}{llll}
\mathrm{N}(25) & -3.1967 & -2.1903 & 0.5841 \\
\mathrm{Se}(26) & -3.5041 & -2.1965 & -1.2438 \\
\mathrm{~N}(27) & -4.3168 & -3.8139 & -0.8411 \\
\mathrm{C}(28) & -3.7179 & -4.2957 & 0.1462 \\
\mathrm{C}(29) & -3.0784 & -3.3964 & 0.9022 \\
\mathrm{C}(30) & -8.8211 & -3.6482 & -1.5992 \\
\mathrm{C}(31) & -7.7186 & -4.4298 & -1.7539 \\
\mathrm{~N}(32) & -6.6108 & -3.8728 & -1.4266 \\
\mathrm{Se}(33) & -6.9817 & -2.3199 & -0.5192 \\
\mathrm{C}(34) & -5.3204 & -4.4917 & -1.6589 \\
\mathrm{C}(35) & -0.9025 & 0.1445 & 4.1451 \\
\mathrm{H}(36) & -6.6890 & 5.5819 & 0.0677 \\
\mathrm{H}(37) & -6.0889 & 5.0116 & -1.5576 \\
\mathrm{H}(38) & -8.9157 & 5.3322 & -1.0488 \\
\mathrm{H}(39) & -10.7008 & 3.4344 & -1.3232 \\
\mathrm{H}(40) & -3.4962 & 3.6362 & 2.7404 \\
\mathrm{H}(41) & -5.1276 & 5.2697 & 1.6831 \\
\mathrm{H}(42) & -11.8254 & 1.5075 & -0.5033 \\
\mathrm{H}(43) & -12.6975 & -0.7209 & -0.7147 \\
\mathrm{H}(44) & -11.3260 & -2.5370 & -1.0504 \\
\mathrm{H}(45) & -7.9785 & 0.0212 & -1.3793 \\
\mathrm{H}(46) & -4.2171 & 0.0674 & 0.0624 \\
\mathrm{H}(47) & -1.6018 & -1.9176 & 2.6946 \\
\mathrm{H}(48) & -2.0820 & 2.1003 & 3.0294 \\
\mathrm{H}(49) & -3.8017 & -5.3703 & 0.3870 \\
\mathrm{H}(50) & -2.5152 & -3.6364 & 1.8517 \\
\mathrm{H}(51) & -9.8658 & -3.9453 & -1.7776 \\
\mathrm{H}(52) & -7.6337 & -5.4690 & -2.0688 \\
\mathrm{H}(53) & -5.1269 & -4.2980 & -2.7044 \\
\mathrm{H}(54) & -5.3427 & -5.5380 & -1.3861 \\
\mathrm{H}(55) & -0.9170 & -0.8510 & 4.6808 \\
\mathrm{H}(56) & -1.3084 & 0.7897 & 5.0186 \\
\mathrm{H}(57) & 0.1600 & 0.4116 & 3.8882
\end{array}
$$




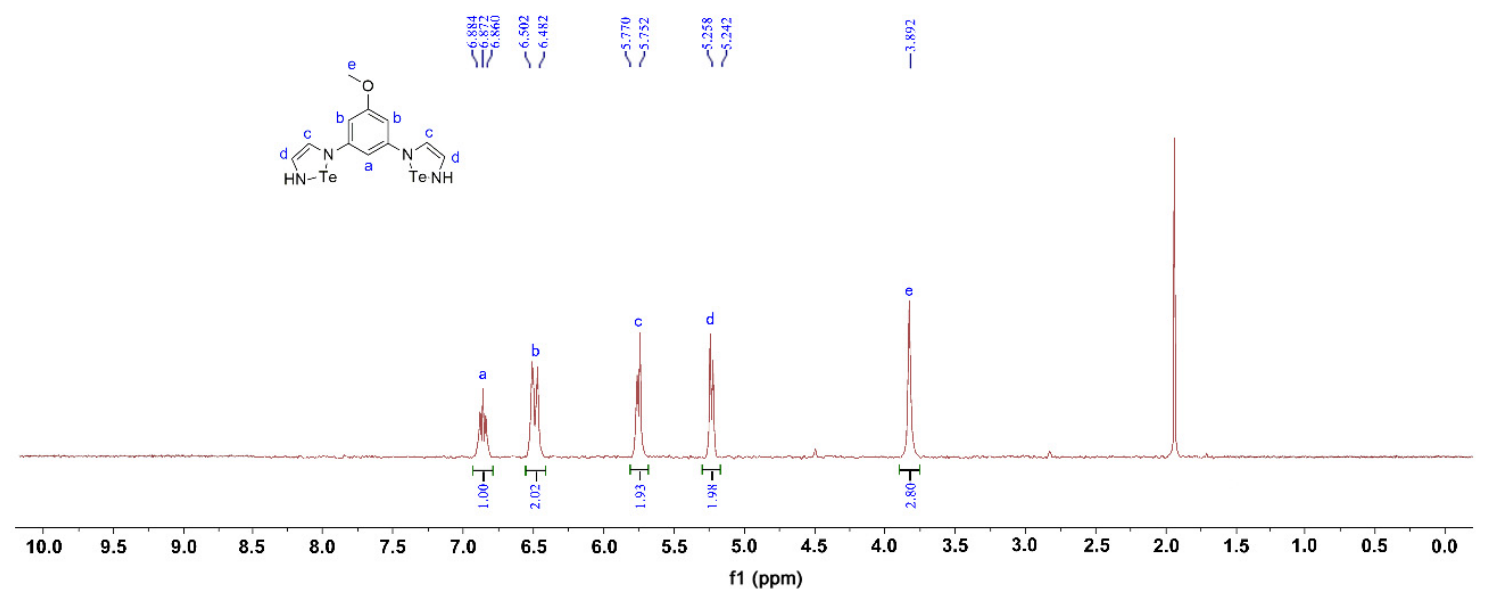

Figure S14. ${ }^{1} \mathrm{H}$ NMR spectra (400 MHz) of 1,3-bis(1,2,5-telluradiazol-1-yl)anisole in $\mathrm{CD}_{3} \mathrm{CN}$.

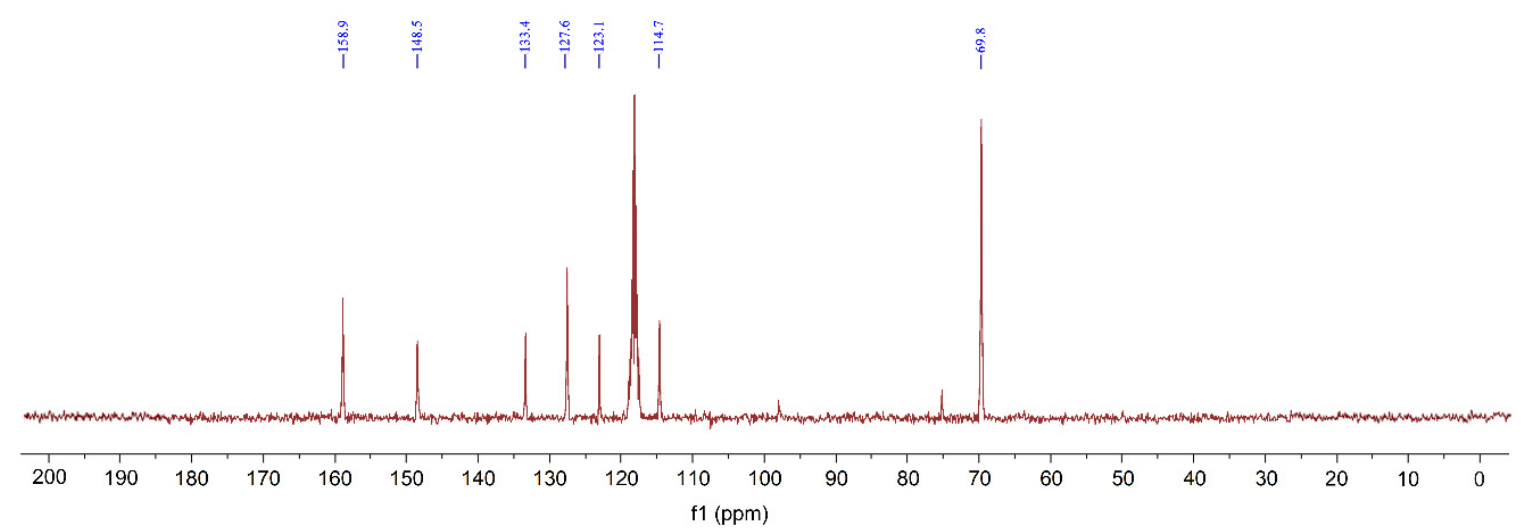

Figure S15. ${ }^{13} \mathrm{C}$ NMR spectra (100 MHz) of 1,3-bis(1,2,5-telluradiazol-1-yl)anisole in $\mathrm{CD}_{3} \mathrm{CN}$. 


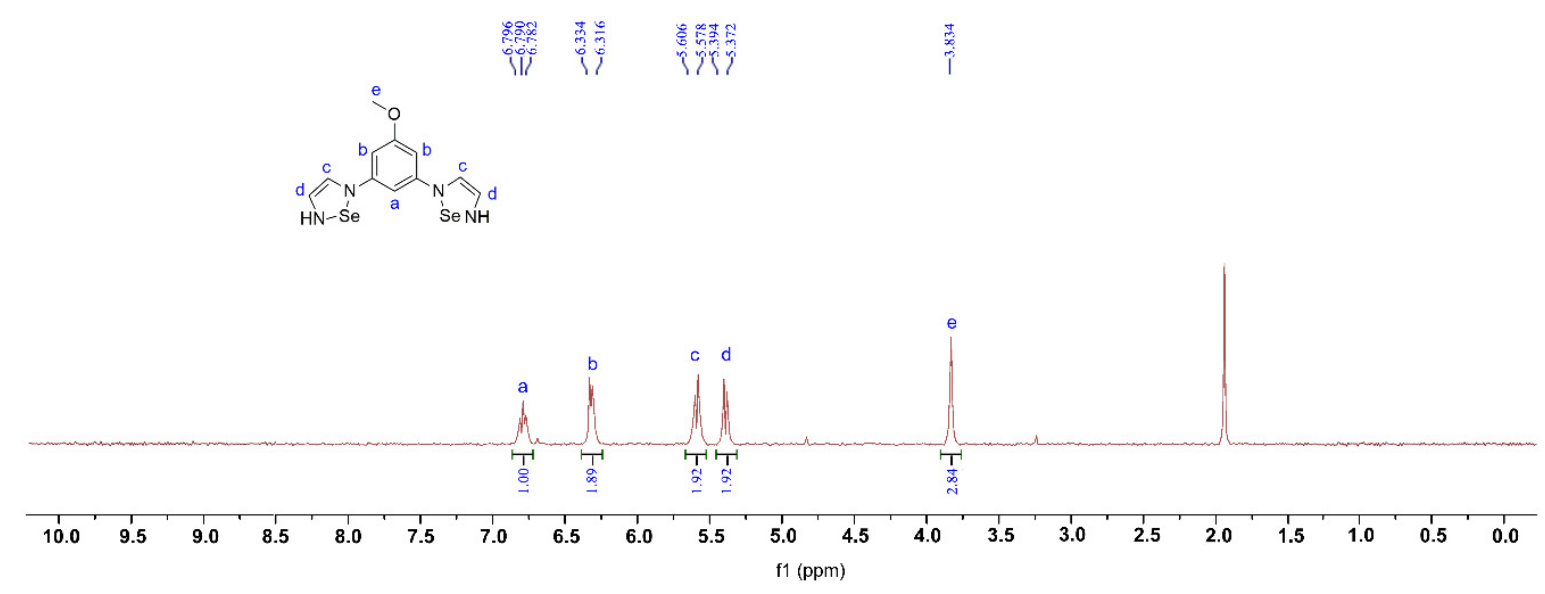

Figure S16. ${ }^{1} \mathrm{H}$ NMR spectra (400 MHz) of 1,3-bis(1,2,5-selenadiazol-1-yl)anisole in $\mathrm{CD}_{3} \mathrm{CN}$.

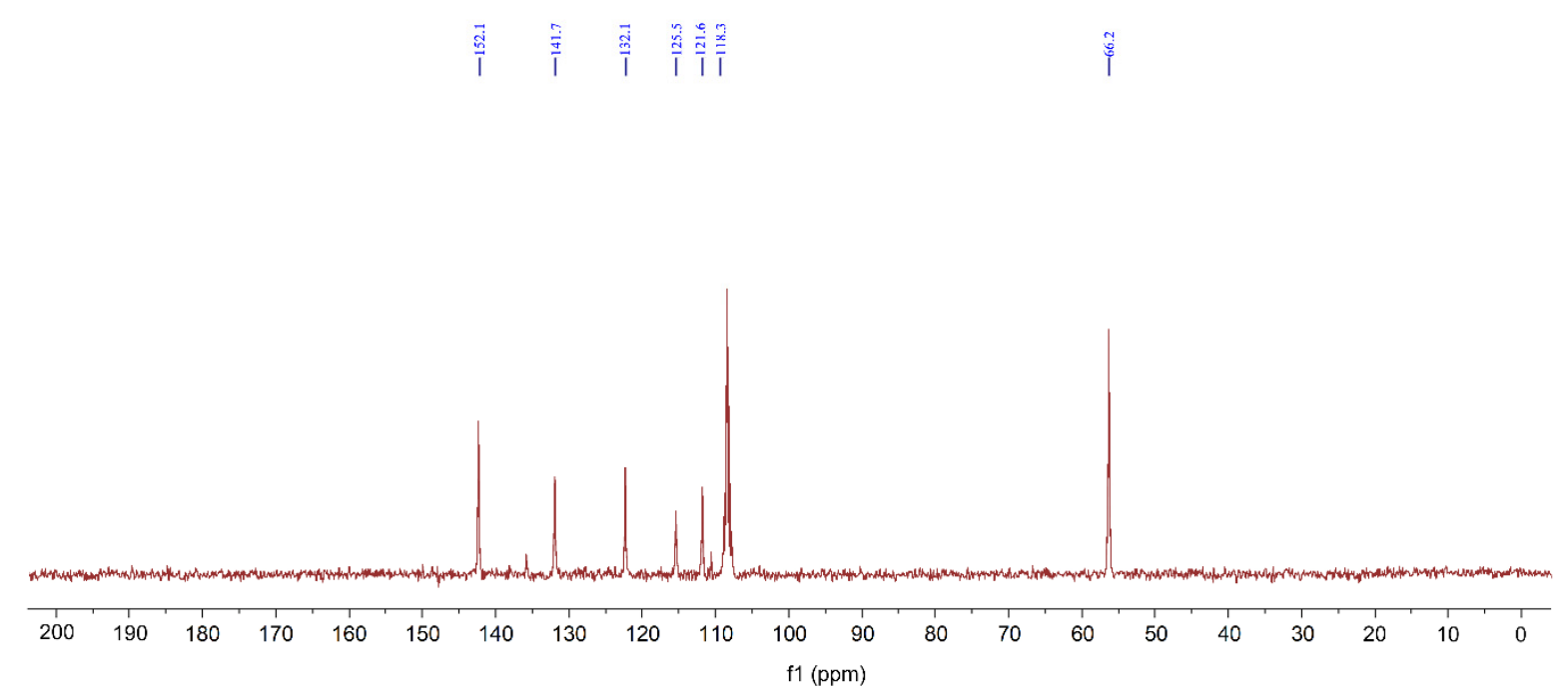

Figure S17. ${ }^{13} \mathrm{C}$ NMR spectra $(100 \mathrm{MHz})$ of 1,3-bis(1,2,5-selenadiazol-1-yl)anisole in $\mathrm{CD}_{3} \mathrm{CN}$. 


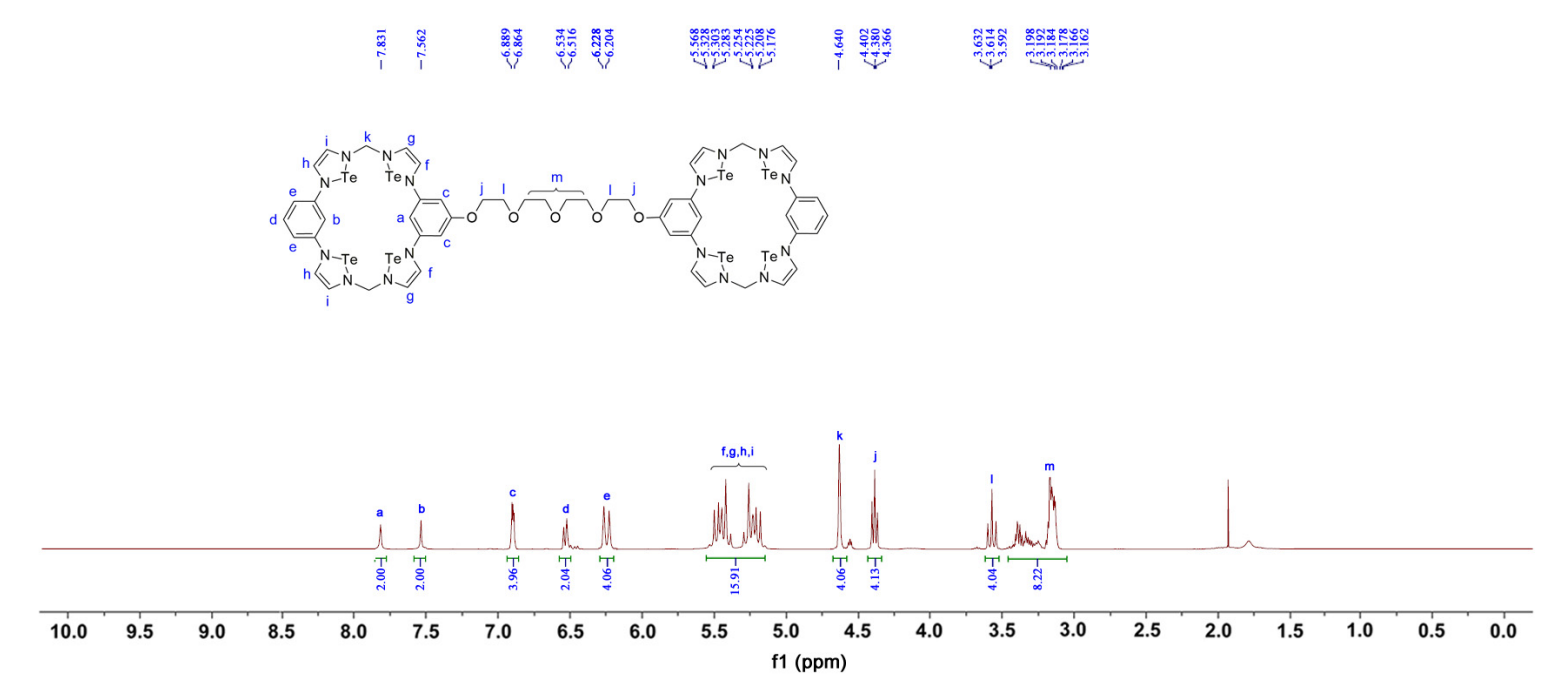

Figure S18. ${ }^{1} \mathrm{H}$ NMR spectra (400 MHz) of homoditopic tellura[4]arene macrocyclic receptor $(\mathrm{TeA}[4])$ in $\mathrm{CD}_{3} \mathrm{CN}$.

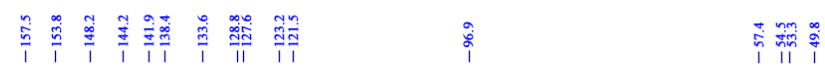

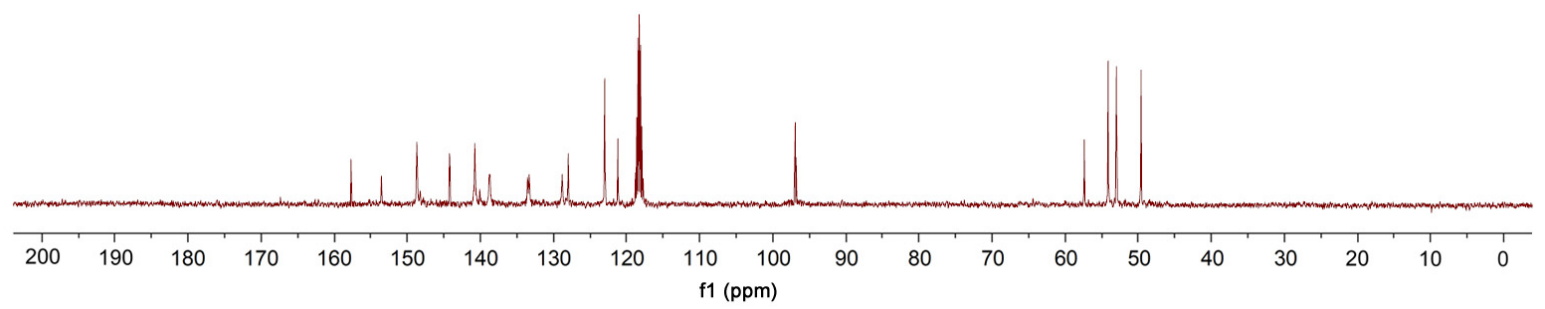

Figure S19. ${ }^{13} \mathrm{C}$ NMR spectra $(100 \mathrm{MHz})$ of homoditopic tellura[4]arene macrocyclic receptor $(\mathrm{TeA}[4])$ in $\mathrm{CD}_{3} \mathrm{CN}$. 

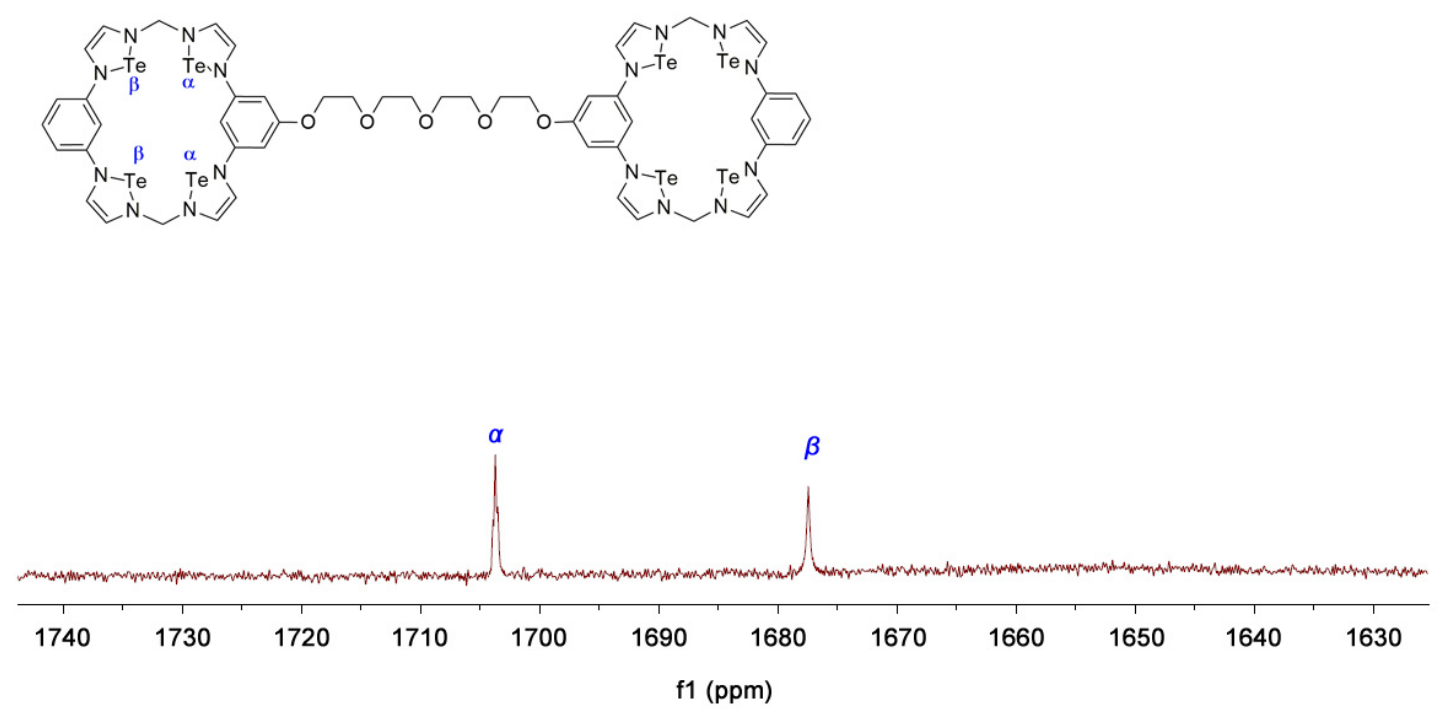

Figure S20. ${ }^{125} \mathrm{Te}$ NMR spectra $(252.4 \mathrm{MHz})$ of homoditopic tellura[4]arene macrocyclic receptor $(\mathrm{Te} A[4])$ in $\mathrm{CD}_{3} \mathrm{CN}$.

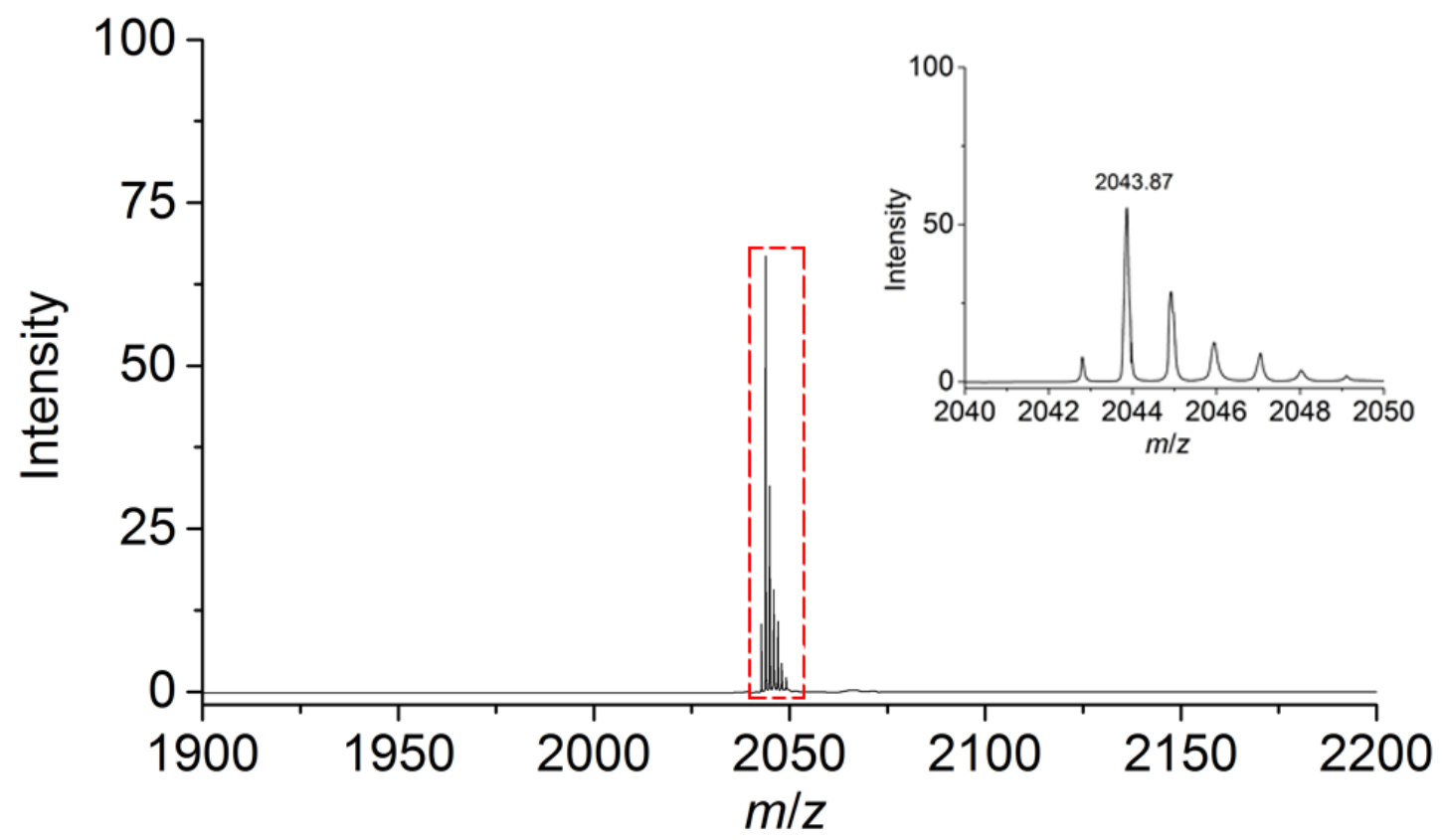

Figure S21. ESI-MS data of TeA[4] macrocyclic receptor. 


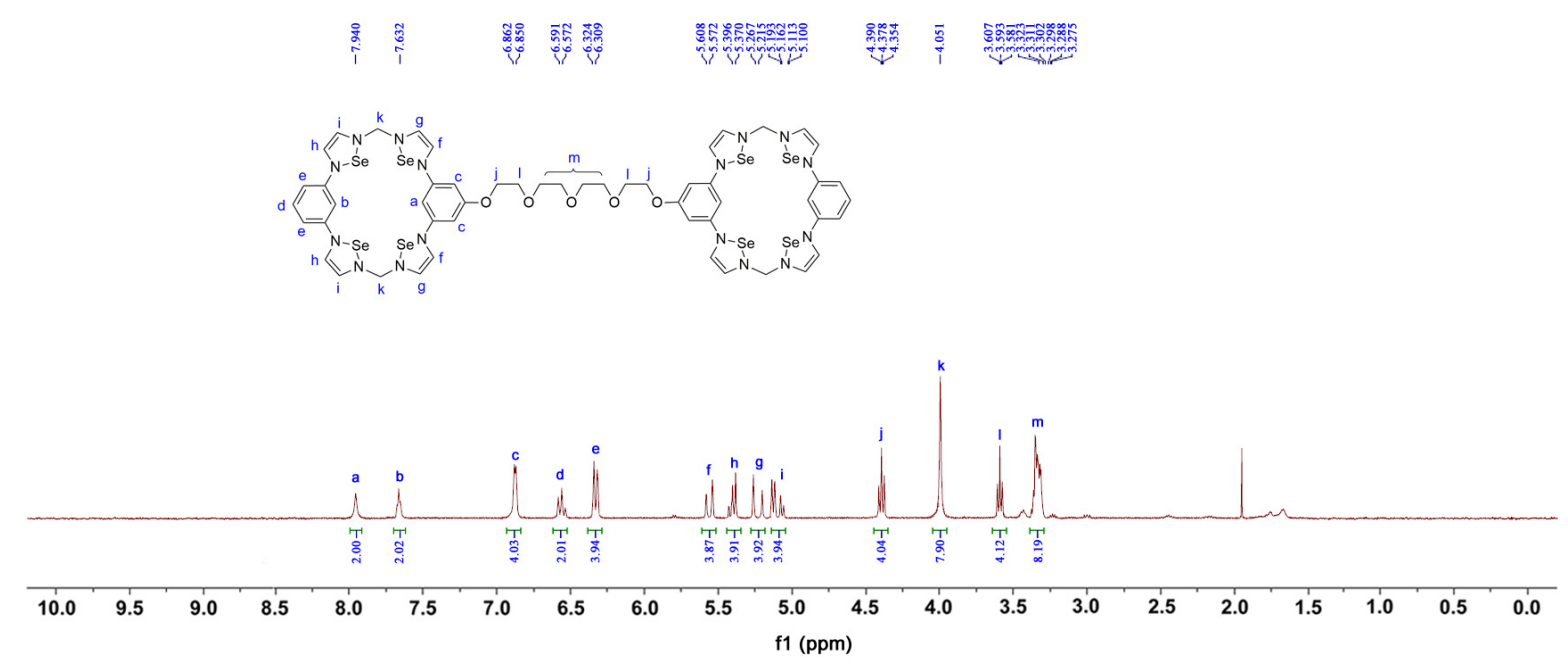

Figure S22. ${ }^{1} \mathrm{H}$ NMR spectra (400 MHz) of homoditopic selena[4]arene macrocyclic receptor $(\mathrm{SeA}[4])$ in $\mathrm{CD}_{3} \mathrm{CN}$.
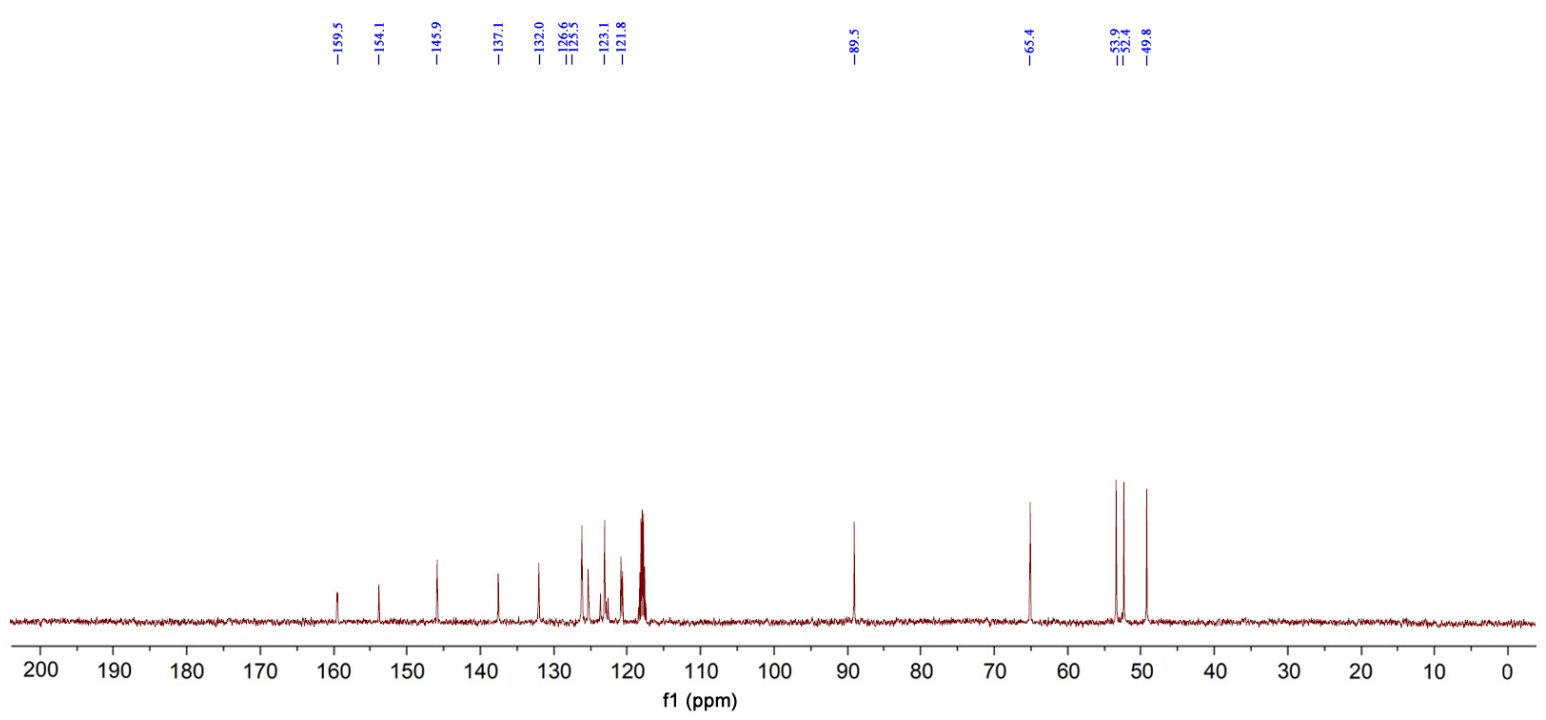

Figure S23. ${ }^{13} \mathrm{C}$ NMR spectra $(100 \mathrm{MHz})$ of homoditopic selena[4]arene macrocyclic receptor $(\mathrm{SeA}[4])$ in $\mathrm{CD}_{3} \mathrm{CN}$. 

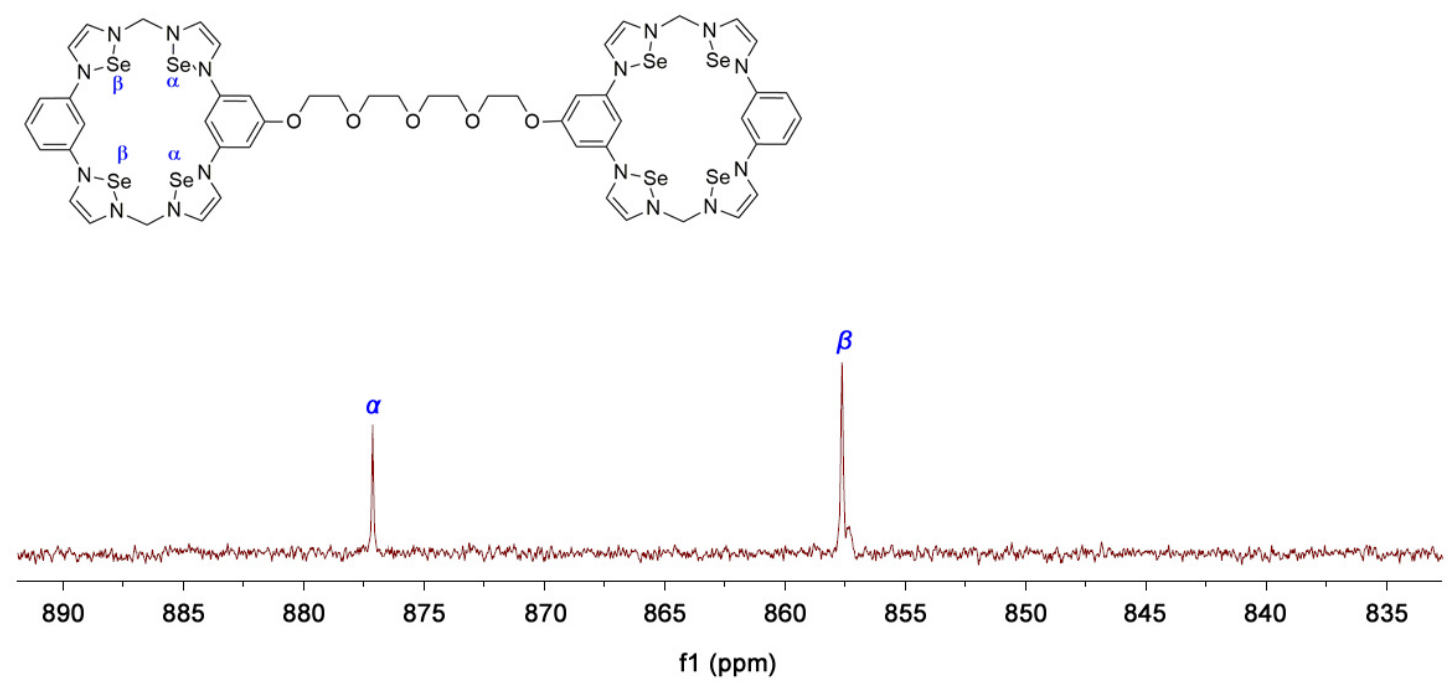

Figure S24. ${ }^{77} \mathrm{Se}$ NMR spectra $(152.6 \mathrm{MHz})$ of homoditopic selena[4] arene macrocyclic receptor $(\mathrm{SeA}[4])$ in $\mathrm{CD}_{3} \mathrm{CN}$.

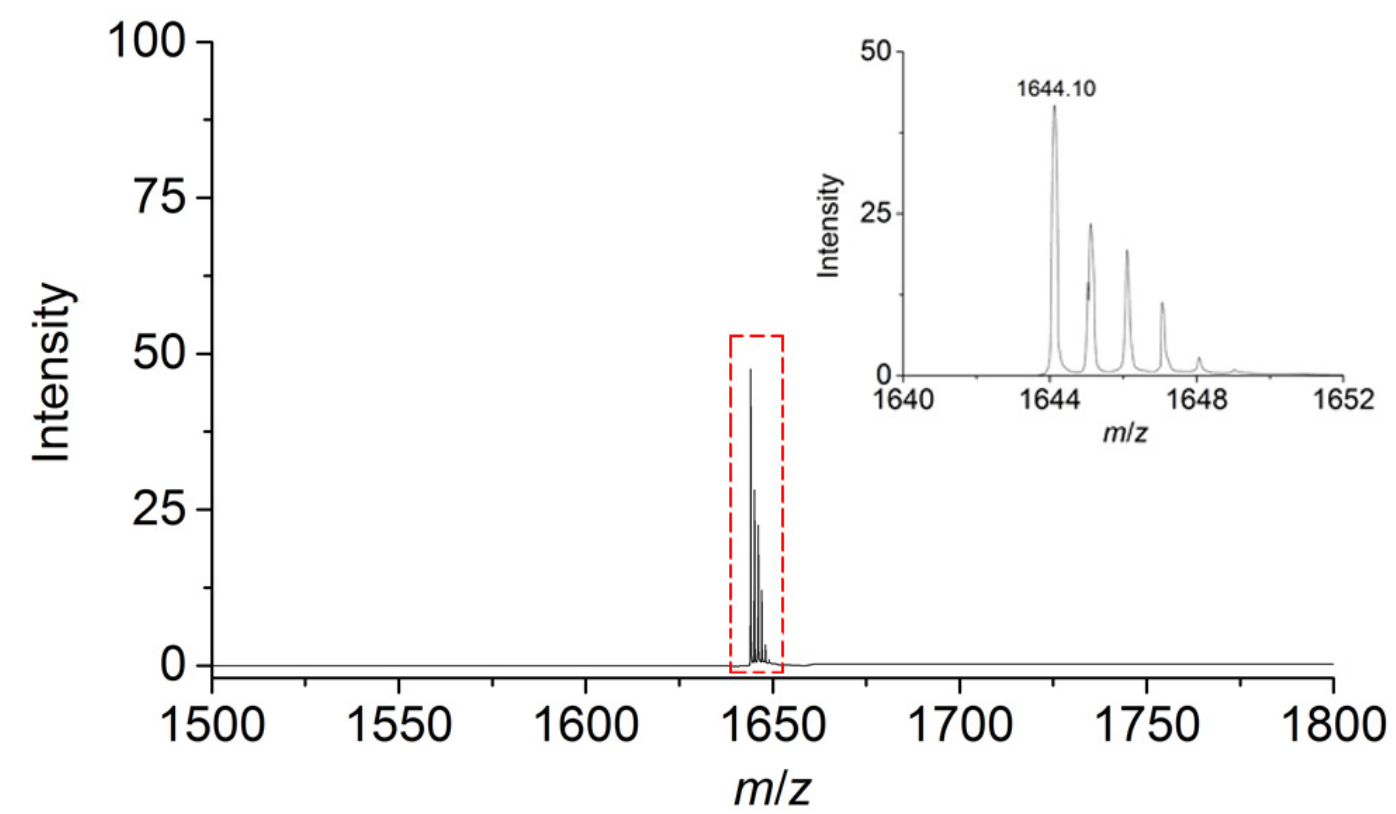

Figure S25. ESI-MS data of SeA[4] macrocyclic receptor.

\section{References}

(1) Carothers, C. H. Trans. Faraday Soc. 32, 39-53 (1936).

(2) Niu, Z. B., Huang, F. H. \& Gibson, H. W. J. Am. Chem. Soc. 133, 2836-2839 (2011). 
(3) Marshall, A. G. Biophysical Chemistry: Principles, Techniques, and Applications; pp 7077, John Wiley \& Sons: New York (1978). 